\title{
Antibiotic prophylaxis in cesarean sections: a tertiary care hospital based survey
}

\author{
Sneha Mishra, Aastha Raheja, Krishna Agarwal*, Gauri Gandhi
}

Department of Obstetrics and Gynecology, Maulana Azad Medical College, New Delhi, India

Received: 02 April 2021

Accepted: 05 May 2021

\section{*Correspondence:}

Dr. Krishna Agarwal,

E-mail: drkrishna.agarwal@gmail.com

Copyright: (C) the author(s), publisher and licensee Medip Academy. This is an open-access article distributed under the terms of the Creative Commons Attribution Non-Commercial License, which permits unrestricted non-commercial use, distribution, and reproduction in any medium, provided the original work is properly cited.

\begin{abstract}
Background: The objective of the study was to study the pattern of prophylactic antibiotics usage in caesarean sections in Indian settings.

Methods: A cross-sectional observational study was done on women undergoing elective and emergency caesarean sections in the Department of obstetrics and gynecology who were given antibiotics according to the existing trends in the hospital.

Results: Almost $72 \%$ women received prophylactic antibiotics within 30-60 minutes of skin incision while rest $28 \%$ received it before 60 minutes. In post-operative period around $80 \%$ of the women received injectable antibiotics for 48 hours, $12 \%$ for 72 hours and rest $8 \%$ received antibiotics for more than 72 hours. $90 \%$ of the patients received injections ceftriaxone $1 \mathrm{gm}$ IV BD, gentamycin $80 \mathrm{mg}$ IV BD metronidazole $400 \mathrm{mg}$ iv TDS while $10 \%$ received injection Ampicillin $500 \mathrm{mg}$ QID along with Injection Metronidazole $400 \mathrm{mg}$ iv TDS and injection gentamycin $80 \mathrm{mg}$ IV BD. Two percent of the cases developed wound sepsis and required change to higher antibiotics.

Conclusions: In spite of recommendations by International Guidelines for single dose of prophylactic antibiotics, multiple doses are being given. There are no Indian guidelines for antibiotic prophylaxis in cesarean sections and as a result, various combinations of antibiotics are being given for variable duration leading to antibiotic resistance and increased cost of treatment.
\end{abstract}

Keywords: Antibiotics, Prophylactic antibiotics, Cesarean section, Indian settings

\section{INTRODUCTION}

Bacterial infections around the time of childbirth are among the leading causes of maternal mortality worldwide and account for about one-tenth of the global burden of maternal death. ${ }^{1,2}$ Several factors have been associated with increased risk of maternal peripartum infections, including pre-existing maternal conditions (examplemalnutrition, diabetes, obesity, severe anaemia, bacterial vaginosis) and conditions during labour and childbirth (example- prolonged rupture of membranes, multiple vaginal examinations, manual removal of the placenta, caesarean section). Caesarean section is the most important risk factor for maternal infection in the immediate postpartum period. ${ }^{3}$ One of the main strategies to prevent post-caesarean infections includes use of prophylactic antibiotics. ${ }^{4}$. The WHO guideline panel made strong recommendations regarding the administration of prophylactic antibiotics 30-60 minutes before skin incision for women undergoing elective or emergency caesarean section..$^{5}$

Evidence suggests that a single dose of first-generation cephalosporin or penicillin should be used in preference to other classes of antibiotics, particularly because these are broad spectrum antibiotics and widely available in all settings. However, the global use of prophylactic antibiotics for caesarean births varies largely between hospitals, in part because of lack of institutional protocols and uncertainties about the antibiotic regimen of choice 
and correct timing of administration. This survey is being planned to assess the pattern of prophylactic antibiotic use in caesarean sections in our tertiary care hospital in Indian settings.

\section{METHODS}

It was a cross-sectional observational study conducted in a tertiary care Centre at New Delhi. Study population included women undergoing elective and emergency caesarean section in all the units of Department of Obstetrics and Gynecology from January 2018 to June 2018.

The inclusion criterion was any women undergoing emergency or elective caesarean section for any indication. The following women were excluded from the study:

Women with evidence of infections- fever, leukocytosis. Women already on antibiotic therapy. Severe anemia. Associated medical illness. Women who were referred from other hospital after onset of labor

Because of constrain of time, a sample size of convenience was taken. 100 women with valid indication for caesarean section were enrolled in the study. A written informed consent was taken from the patient and they were provided with a patient information sheet. A survey sheet was prepared to obtain all the necessary information. The women were observed in the postpartum period till discharge. All the information obtained was recorded in the predetermined format.

\section{Statistical analysis}

A simple grid was prepared to collate the data in the survey sheet and proportion of responses for each question was calculated.

\section{Ethical approval}

The study was approved by institutional ethics committee, Maulana Azad Medical College, New Delhi, India.

\section{RESULTS}

A total of 100 women who underwent caesarean section for any indication were enrolled in the study group.

The mean age of participants was 24 years. Most of the subjects in the study group belonged to upper lower socioeconomic status by modified Kuppuswami scale. Most of the women undergoing caesarean section were second gravida.

Around $72 \%$ women received the prophylactic antibiotic in the prescribed timing of 30-60 minutes before the skin incision. The only factor found associated with patient not receiving antibiotics within the prescribed time limit was delay during shifting of patient and availability of operation theatre.

Table 1: Demographic and clinical details by groups.

\begin{tabular}{|ll|}
\multicolumn{1}{|l|}{} & $\begin{array}{l}\text { Study group } \\
(\mathbf{n = 1 0 0 )})\end{array}$ \\
\hline Age (years) & $23.69 \pm 2.59$ \\
\hline Socioeconomic class & \\
\hline Lower & 25 \\
\hline Upper lower & 54 \\
\hline Lower middle & 14 \\
\hline Upper middle & 7 \\
\hline Upper & 0 \\
\hline Parity & \\
\hline Primigravida & 33 \\
\hline $2^{\text {nd }}$ gravida & 47 \\
\hline $3^{\text {rd }}$ gravida & 18 \\
\hline$>$ 3rd gravid & 2 \\
\hline
\end{tabular}

Table 2: Pattern of antibiotic usage.

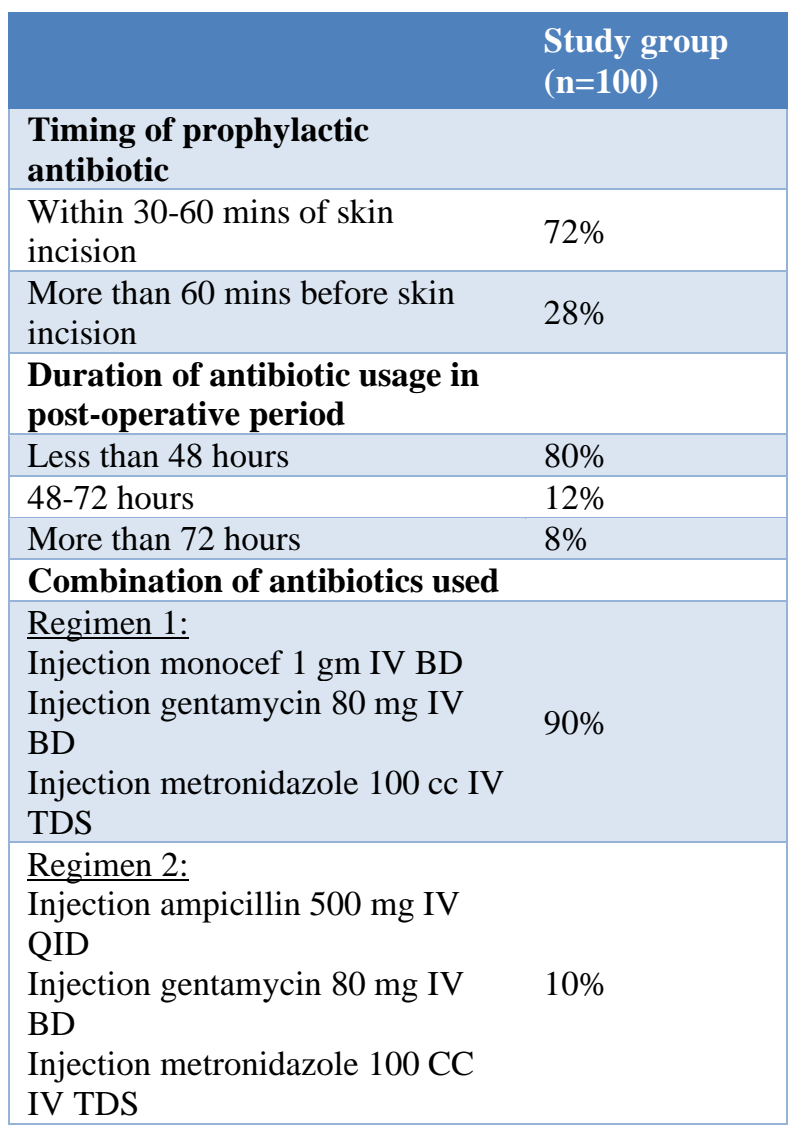

Most of the patients in the study received Triple antibiotics regimen as per the Institutional protocol for up to 48 hours or more. In all cases, Injectable antibiotics were followed by oral antibiotics for 5-6 days. The factors found associated with prolonged antibiotics usage were: surgeon's discretion and patient developing fever/ infections in post-operative period. 
International guidelines do not recommend usage of triple antibiotics for prophylaxis. A single dose of firstgeneration cephalosporin is recommended 30-60 minutes before skin incision.

\section{DISCUSSION}

In our study, all 100 women received prophylactic antibiotic which is as per the recommendations. ${ }^{4}$ Of these women, almost $72 \%$ women received prophylactic antibiotics within 30-60 minutes of skin incision while rest $28 \%$ received it before 60 minutes. Though the majority of women received it within the recommended time limit, it was observed that the time lag while shifting the patient to operation theatre was one of the major causes of not following the recommended norm.

The international guidelines recommend a single dose of antibiotics and there are not enough guidelines for antibiotic usage in developing countries like India. ${ }^{4}$ In our study in post-operative period around $80 \%$ of the women received injectable antibiotics for 48 hours, $12 \%$ for 72 hours and rest $8 \%$ received antibiotics for more than 72hours. The factors found associated with prolonged antibiotics usage were Surgeon's discretion and development of fever/ infections in post-operative period. Since the patients with failure of antimicrobial prophylaxis received 'empiric' treatment, antibiotic culture sensitivity testing is recommended prior to introducing a change in the antimicrobial treatment regimen, to exclude antibiotic resistance. $^{6}$

A total $90 \%$ of the patients received injections ceftriaxone $1 \mathrm{gm} \mathrm{I} / \mathrm{V}$ BD, gentamycin $80 \mathrm{mg}$ IV BD metronidazole 400 mg IV TDS while 10\% received injection Ampicillin 500 mg QID along with Injection Metronidazole $400 \mathrm{mg}$ IV TDS and injection gentamycin 80mg iv BD.

The most frequent microbes isolated from endometrial cultures of women with post-caesarean wound infections are staphylococci, enterococci, anaerobes and ureaplasmas. ${ }^{7}$ Furthermore, when specifically identified, ureaplasma (or Mycoplasma genus) is the most common organism isolated from the amniotic fluid and chorioamnion at caesarean delivery, and is associated with a 3- to 8-fold increased risk of post-caesarean endometritis or wound infection. ${ }^{8,9}$ Bacterial vaginosis is also associated with an increased risk of post-caesarean endometritis. Therefore, the recommended regimen of ceftriaxone alone does not cover frequent isolates or risk factors of such infection. The use of first-generation cephalosporins such as cefazolin provides antibiotic activity against species of Ureaplasma and Mycoplasma but may cause an increase in resistant organisms like anaerobes. Hence, there is a rationale for adding agents such as metronidazole, clindamycin or azithromycin to extend the cover.

There is paucity of studies from developing countries including India that have elucidated the appropriate prophylactic antibiotic regimen in cesarean sections. No guidelines are hence being followed in Indian hospital settings leading to irrational and inappropriate use of antibiotics that are expensive, have side effects and contribute to antibiotic resistance. Our study has special relevance in Indian settings where a large number of cesarean sections are being done every day.

The major limitation of our study is small sample size and observational nature of our study. A randomized trial comparing single versus multiple doses of antibiotics in women undergoing cesarean sections can provide information regarding non-inferiority of one regimen over other. It can also help in shaping of indigenous guidelines as per Indian population.

\section{CONCLUSION}

In spite of recommendations by International Guidelines for single dose of prophylactic antibiotics, multiple doses are being given. There are no Indian guidelines for antibiotic prophylaxis in cesarean sections and as a result, various combinations of antibiotics are being given for variable duration leading to antibiotic resistance and increased cost of treatment.

Hence, there is a need for further studies for prophylactic antibiotics usage for caesarean sections in low-income developing countries.

\section{Funding: No funding sources \\ Conflict of interest: None declared}

Ethical approval: The study was approved by the Institutional Ethics Committee

\section{REFERENCES}

1. Say L, Chou D, Gemmill A, Tuncalp O, Moller AB, Daniels $\mathrm{J}$ et al. Global causes of maternal death: a WHO systematic analysis. Lancet Global Health. 2014; 2:e323-33.

2. Khan KS, Wojdyla D, Say L, Gulmezoglu AM, Van Look PF. WHO analysis of causes of maternal death: a systematic review. Lancet.2006;367:1066-74.

3. Gibbs RS. Clinical risk factors for puerperal infection. ObstetGynecol .1980;55:18S-4S.

4. Bastu E, Gulmezoglu AM. Antibiotic prophylaxis versus no prophylaxis for preventing infection after caesarean section: RHL commentary. The WHO Reproductive Health Library; Geneva: World Health Organization.

5. WHO Recommendations for Prevention and Treatment of Maternal Peripartum Infections. Geneva: World Health Organization. 2015.

6. Liu R, Lin L, Wang D. Antimicrobial prophylaxis in caesarean section delivery. Exp Ther Med. 2016;12(2):961-4.

7. Emmons SL, Krohn M, Jackson M, Eschenbach DA. Development of wound infections among women 
undergoing cesarean section. Obstet Gynecol. 1988;72:559-64.

8. Roberts S, Maccato M, Faro S, Pinell P. The microbiology of post-cesarean wound morbidity. Obstet Gynecol. 1993;81:383-6.

9. Andrews WW, Shah SR, Goldenberg RL, Cliver SP, Hauth JC, Cassell GH. Association of post-cesarean delivery endometritis with colonization of the chorioamnion by Ureaplasma urealyticum. Obstet Gynecol. 1995;85:509-14.

Cite this article as: Mishra S, Raheja A, Agarwal K, Gandhi G. Antibiotic prophylaxis in cesarean sections: a tertiary care hospital based survey. Int J Reprod Contracept Obstet Gynecol 2021;10:2221-4. 